\title{
Early impact of fixed orthodontic therapy on gingival health status in relation to weight status
}

\author{
Zaid Ali Alasadi B.D.S. (1) \\ Alhan Ahmed Qasim B.D.S, M.Sc. ${ }^{(2)}$
}

\begin{abstract}
Background: fixed orthodontic appliances deleterious influence on gingival health is well documented. Association between weight status and gingival health is presented in many studies. This study aimed to evaluate how early the impact of fixed orthodontic therapy on patients ' gingival health, and if there are differences of that impact among different weight status groups.

Materials and Methods: Sample consisted of 54 patients (25 males, 29 females; age limits are $16-18$ years) going under the course of treatment with fixed orthodontic appliance. Patients were categorized according to their Body Mass Index (BMI) into 3 weight status groups considering WHO charts in 2007 (underweight, normal weight, overweight and obese), then determination of each patient's gingival health status was through the criteria of the gingival index (GI) by Loe and Silness in 1963 which modified by Loe in 1967. Records of gingival index for all patients who met specific criteria were taken in three time points [before bonding (1st visit), 2 weeks after bonding (2nd visit), and 4 weeks after bonding (3rd visit)]. Also BMI of the patients were checked at each of the three visits.

Results: There was a significant increase in gingival index for all BMI weight status groups after just two weeks of treatment, and the increase continues during the $3^{\text {rd }}$ visit, with no significant difference in impact among weight status groups.

Conclusions: oral health preventive measures should be applied rapidly and equally to all patients treated with fixed orthodontic appliances, without taking their BMI weight status in consideration.

KEY WORDS: early, fixed, orthodontic, gingival health, weight, BMI. (Received: 12/8/2018; Accepted: 16/9/2018)
\end{abstract}

\section{INTRODUCTION}

Fixed orthodontic appliances introduce an additional constituent to the oral cavity complex that may enhance oral environment in a variety of ways ${ }^{(1)}$. On the other hand, Orthodontic treatment leads to changes in the oral environmental factors that encourage deteriorations in oral hygiene status and increased plaque coverage in orthodontic patients to 2 or 3 folds than levels observed in high plaque forming subjects without appliance ${ }^{(2)}$. Elevation of plaque and calculus presence, and increased gingival inflammation are all factors supplement the risk of caries activity and periodontal health, and shake the stability of the oral environment ${ }^{(3)}$. Pain is considered one of the major factors that cause deterioration in gingival health status, due to difficulties in performing usual preventive measures (4) and changes in patients` dietary habits ${ }^{(5)}$. Pain and discomfort caused by fixed orthodontic appliance can last for 14 days (6). The World Health Organization (WHO) describes Obesity as one of today's most neglected public health problem, affecting every region of the globe ${ }^{(7)}$.

(1) Ministry of health, Baghdad, Iraq

(2) B.D.S, M.Sc., Assistant Professor, Department of Pedodontics and Preventive Dentistry, College of Dentistry, University of Baghdad.
Well documented researches had established significant relationship between weight status and periodontal diseases $(8,9)$. Gingivitis, the mildest form of periodontal disease, is a rapidly inducible and reversible inflammatory affection of the gingiva, mainly caused by accumulation of bacterial biofilm. The combination of bacterial infection and persistent inflammatory response can eventually induce the progressive destruction of the deeper periodontal tissues, a worse form of periodontal disease called periodontitis (3). Gingivitis and periodontitis can be considered a continuum of the same inflammatory process, although it is important to note that gingivitis lesions do not necessarily progress to periodontitis ${ }^{(10)}$. Additional risk factors include genetic susceptibility, tobacco smoking, alcohol consumption, and systemic conditions such as diabetes, osteoporosis, malnutrition, and overweight and obesity can facilitate the progression of gingivitis into periodontitis (11-13). Since there is a well known effect of fixed orthodontic appliance on gingival health that is mainly provoked by pain. And previous studies findings that correlate periodontal problems to malnourishment and obesity. The hypothesis of the present study is that there will be an early impact of fixed orthodontic appliance on gingival health, and this impact will be of different effect on different weight status groups. Literature had showed only one study concerning differences of effects of fixed orthodontic treatment on gingival 
health status on patients with different BMI weight statuses, which is a study by Von Bremen et al ${ }^{(14)}$

\section{MATERIALS AND METHODS}

\section{Study group}

This was an observational, prospective, selfcontrolled study. The study group consisted of patients about to be orthodontically treated with fixed appliances in the Department of Orthodontics inside the specialized dental center for prosthodontics and orthodontics in BabAlmoadham in Baghdad/Iraq. The inclusion criteria was only to be within the age limits, while the exclusion criteria were: (1) subjects with any systemic or oral diseases or any kind of allergies (2) subjects undergone orthodontic treatment before (3) subjects with congenital craniofacial anomalies (4) subjects with any oral or general pain (5) subjects who had been; or willing to be on a diet (include fasting) (6) subjects require the usage of space gaining appliance during their treatment (7) subjects receiving any medications (8) subjects with any kind of addiction (include all types of smoking) (9) uncooperative patients who didn't commit to the previous criteria during the study period, or miss appointments in \pm 1 day. Since the present study is a follow up study, In order to minimize the effects of different known and unknown variables, subjects before wearing orthodontic appliances were used as a study control. Informed consent was obtained from all participants and their parents after a detailed explanation of the study. Approval of the study protocol was obtained by the scientific committee of the pediatric and prevention department/Collage of dentistry/University of Baghdad.

Due to the dental center policy, all patients must receive a full dental treatment, plus a complete scaling and polishing treatment and oral hygiene instructions, maximally 2 days prior starting the bonding of orthodontic fixed appliance. All patients were orthodontically treated using the same equipments [Stainless steel arch wire (World class tech. /Ortho classic / USA), Stainless steel brackets (Stratus / Fairfield / USA), Bonding agent, (Resilience / ortho technology / USA), Stainless steel bands (World class tech. /Ortho classic / USA), Luting glass ionomer cement (Riva luting /SDI /Ireland), Ligature rings (World class tech./Ortho classic / USA)].

At the beginning of each of the three visits; a data sheet were filled for each subject; including records of height and weight taken for each patient in order to calculate their body mass index (BMI) ${ }^{(15)}$, next, according to the results of those calculations, patients were grouped under three categories (underweight, normal weight, overweight and obese), considering a standerd charts set by world health organization (WHO); specific for each age (in months) and gender ${ }^{(16)}$. The final number of patients in each category was (underweight group $=16$, normal weight group $=25$, overweight and obese group $=13$ )

\section{Oral examination}

Clinical examination and oral hygiene assessment have been done on dental chairs of the orthodontic department of the specialized dental center of Bab-Almuadham, fallowing standardized conditions of the basic methods of oral health surveys set by WHO in $1997^{(17)}$. A complete oral examination to all aspects and surfaces of soft and hard tissues was done for each participant, and all observations were recorded on each patient's case sheet. The assessment of gingival health of the patients was done through examining four aspects (facial, lingual or palatal, mesial, distal) of all teeth according to the criteria of the gingival index (GI), which was set by Loe and Silness ${ }^{(18)}$, and modified later by Loe ${ }^{(19)}$.

\section{Statistical Analysis}

Residuals are tested with the use of Shapiro-wilk test of normality after applying it to the whole sample, and all appeared to be normally distributed. General linear model (GLM) for repeated measures was used to determine the different readings of the variables included in the study for the same subject in different time intervals for both (within-subjects factors and between-subjects factors), also using of Bonferroni because of having three levels in the study structure, so Univariate tests were illustrated to show the within-subjects effects, while Pairwise t-test comparisons were used in estimation of means difference between each two visits. One-way ANOVA was used in order to compare between the three categories that the sample was divided into, in order to test significance of the relations between them. The level of significance was set at 0.05. Statistical analyses were performed with the use of (Statistical Package for Social Science SPSS version 21),

\section{RESULTS}

The distribution (in numbers and percentages) of the sum of study participants inside each BMI 
weight status group in the three visits is shown in table 1. The table reveals that the sum and percentage of patients inside each group did not had any change during the three visits, while table 2 shows that changes in patients' Body Mass Index (BMI) through the three visits were not significant. Table 3 shows descriptive and statistical test of gingival index changes through visits and by weight status. The table shows under the column of "general liner model (GLM) of repeated measures" that there was significant change through the three visits for all weight status groups. Under the column "pairwise t-test" comparing between each two visits, which shows that there was a significant increase in the $2^{\text {nd }}$ visit for all weight status groups, and the increase continues in the $3^{\text {rd }}$ visit. Comparing the readings of the three weight status groups in each visit had been showed in the raw "ANOVA", the results in it shows no significant difference among weight status groups at each visit.

Table 1: Distribution (in numbers and percentages) of the study subjects weight status through the three visits

\begin{tabular}{|c|c|c|c|c|c|c|}
\hline \multirow[t]{2}{*}{ BMI Weight status } & \multicolumn{2}{|c|}{$1^{\text {st }}$ visit } & \multicolumn{2}{|c|}{$2^{\text {nd }}$ visit } & \multicolumn{2}{|c|}{$3^{\text {rd }}$ visit } \\
\hline & Sum & $\%$ & Sum & $\%$ & Sum & $\%$ \\
\hline Underweight & 16 & $29.6 \%$ & 16 & $29.6 \%$ & 16 & $29.6 \%$ \\
\hline Normal weight & 25 & $46.3 \%$ & 25 & $46.3 \%$ & 25 & $46.3 \%$ \\
\hline Overweight and obese & 13 & $24.1 \%$ & 13 & $24.1 \%$ & 13 & $24.1 \%$ \\
\hline Total sum of samples & 54 & $100 \%$ & 54 & $100 \%$ & 54 & $100 \%$ \\
\hline
\end{tabular}

Table 2: Descriptive and statistical test of changes of means of BMI of each weight status group between visits by gender

\begin{tabular}{|c|c|c|c|c|c|c|c|c|c|}
\hline \multirow{2}{*}{ BMI Weight status } & \multirow{2}{*}{ Gender } & \multicolumn{2}{|c|}{$1^{\text {st }}$ visit } & \multicolumn{2}{|c|}{$2^{\text {nd }}$ visit } & \multicolumn{2}{|c|}{$3^{\text {rd }}$ visit } & \multicolumn{2}{c|}{ ANOVA } \\
\cline { 3 - 9 } & & Mean & \pm SD & Mean & \pm SD & Mean & \pm SD & F & Sig \\
\hline \multirow{2}{*}{ Underweight } & Male & 18.34 & $\mathbf{0 . 2 6}$ & $\mathbf{1 8 . 1 4}$ & $\mathbf{0 . 2 8}$ & $\mathbf{1 8 . 2 7}$ & $\mathbf{0 . 1 3}$ & $\mathbf{0 . 8 6 0}$ & $\mathbf{0 . 4 3 9}$ \\
\cline { 2 - 10 } & Female & 17.17 & $\mathbf{1 . 4 9}$ & $\mathbf{1 7 . 0 5}$ & $\mathbf{1 . 4 7}$ & $\mathbf{1 7 . 1 5}$ & 1.50 & $\mathbf{1 . 0 0 2}$ & $\mathbf{0 . 3 8 1}$ \\
\hline \multirow{2}{*}{ Normal weight } & Male & $\mathbf{2 1 . 1 4}$ & $\mathbf{1 . 7 6}$ & $\mathbf{2 1 . 0 1}$ & $\mathbf{1 . 7 2}$ & $\mathbf{2 1 . 2 2}$ & $\mathbf{1 . 6 4}$ & $\mathbf{2 . 5 2 1}$ & $\mathbf{0 . 1 1 0}$ \\
\cline { 2 - 9 } & Female & $\mathbf{1 9 . 9 0}$ & $\mathbf{2 . 2 7}$ & $\mathbf{1 9 . 7 4}$ & $\mathbf{2 . 3 2}$ & $\mathbf{1 9 . 7 9}$ & $\mathbf{2 . 3 5}$ & $\mathbf{0 . 7 7 5}$ & $\mathbf{0 . 4 7 3}$ \\
\hline \multirow{2}{*}{ Overweight and obese } & Male & $\mathbf{3 0 . 8 2}$ & $\mathbf{4 . 3 3}$ & $\mathbf{3 0 . 4 8}$ & $\mathbf{4 . 0 1}$ & $\mathbf{3 0 . 0 2}$ & $\mathbf{3 . 2 8}$ & $\mathbf{2 . 6 6 8}$ & $\mathbf{0 . 1 5 0}$ \\
\cline { 2 - 10 } & Female & $\mathbf{2 7 . 5 4}$ & $\mathbf{5 . 0 4}$ & $\mathbf{2 7 . 3 6}$ & $\mathbf{4 . 6 4}$ & $\mathbf{2 7 . 5 4}$ & $\mathbf{5 . 3 6}$ & $\mathbf{0 . 0 5 0}$ & $\mathbf{0 . 8 8 0}$ \\
\hline
\end{tabular}

Table 3: Descriptive and statistical test of gingival index changes through visits and by weight status.

\begin{tabular}{|c|c|c|c|c|c|c|c|c|c|c|}
\hline \multirow[t]{2}{*}{ Weight status } & \multicolumn{2}{|c|}{$1^{\text {st }}$ visit } & \multicolumn{2}{|c|}{$2^{\text {nd }}$ visit } & \multicolumn{2}{|c|}{$3^{\text {rd }}$ visit } & \multicolumn{2}{|c|}{ GLM for repeated measures } & \multicolumn{2}{|c|}{ Pairwise t-test } \\
\hline & $\mathbf{M}$ & $\pm \mathrm{SD}$ & $\mathbf{M}$ & $\pm \mathrm{SD}$ & $\mathbf{M}$ & $\pm \mathrm{SD}$ & $\mathbf{F}$ & $\mathbf{P}$ & visits & $\mathbf{P}$ \\
\hline \multirow[t]{3}{*}{ Underweight } & \multirow{3}{*}{0.06} & \multirow{3}{*}{0.06} & \multirow{3}{*}{0.19} & \multirow{3}{*}{0.18} & \multirow{3}{*}{0.28} & \multirow{3}{*}{0.23} & \multirow{3}{*}{11.950} & \multirow{3}{*}{$0.000^{*}$} & $1^{\text {st }} \times 2^{\text {nd }}$ & 0.012* \\
\hline & & & & & & & & & $\mathbf{1}^{\text {st }} \times 3^{\text {rd }}$ & 0.002* \\
\hline & & & & & & & & & $2^{\text {nd }} \times 3^{\text {rd }}$ & 0.159 \\
\hline \multirow[t]{3}{*}{ Normal weight } & \multirow{3}{*}{0.04} & \multirow{3}{*}{0.05} & \multirow{3}{*}{0.21} & \multirow{3}{*}{0.21} & \multirow{3}{*}{0.36} & \multirow{3}{*}{0.33} & \multirow{3}{*}{19.353} & \multirow{3}{*}{$0.000^{*}$} & $1^{\text {st }} \times 2^{\text {nd }}$ & 0.000* \\
\hline & & & & & & & & & $\mathbf{1}^{\text {st }} \times 3^{\text {rd }}$ & $0.000^{*}$ \\
\hline & & & & & & & & & $2^{\text {nd }} \times 3^{\text {rd }}$ & 0.017* \\
\hline \multirow{3}{*}{$\begin{array}{c}\text { Overweight } \\
\text { \& obese }\end{array}$} & \multirow{3}{*}{0.08} & \multirow{3}{*}{0.10} & \multirow{3}{*}{0.25} & \multirow{3}{*}{0.27} & \multirow{3}{*}{0.44} & \multirow{3}{*}{0.43} & \multirow{3}{*}{9.767} & \multirow{3}{*}{$0.002^{*}$} & $1^{\text {st }} \times 2^{\text {nd }}$ & $\mathbf{0 . 0 3 7}^{*}$ \\
\hline & & & & & & & & & $\mathbf{1}^{\text {st }} \times 3^{\text {rd }}$ & 0.010* \\
\hline & & & & & & & & & $2^{\text {nd }} \times 3^{\text {rd }}$ & 0.128 \\
\hline $\mathbf{F}$ & \multicolumn{2}{|c|}{1.621} & \multicolumn{2}{|c|}{0.359} & \multicolumn{2}{|c|}{0.847} & & & & \\
\hline $\mathbf{P}$ & \multicolumn{2}{|c|}{0.208} & 0.7 & & 0.4 & & & & & \\
\hline
\end{tabular}

$*$ Significant $(\mathrm{p}<0.05)$ 


\section{DISCUSSION}

The present study had the closest design to Von Bremen et al study (14), but still had few differences from it. Its study samples were categorized according to their body mass index into three categories (normal weight, overweight, and obese), also, records had been taken in only two visits (before bonding and after debonding of fixed orthodontic appliance), that is because Von Bremen study was retrospective, depending on previously recorded data and photographs in scoring gingival index. The Von Bremen study has some advantages over the present study, like larger number of the study subjects (181 patients). The Von Bremen et al study had found that there was a significant increase in gingivitis of obese patients than normal and overweight ones. This finding may be considered consecutive with the present study findings, since in this study in overweight and obese group there were only 4 obese patients of 13 , while the rest were just overweight subjects, so the readings of overweight subjects were more influential on the group values than obese subjects. The result of the present study showed no significant difference between weight status groups, this may be referred to four hypothetical reasons: [1] the duration of the study was not long enough to reveal differences among weight status groups, because gingival inflammation is directly related to the duration of treatment (20). [2] The differences in BMI were not enough to provoke any differences on gingival health status, or in other words, weight differences are not effective yet to produce bad effects on gingival health /or deteriorate an already found ones. This can be noticed in Von Bremen et al study ${ }^{(19)}$, when overweight group showed no significant difference while obese group showed significant increase in gingival index. [3] The effect of fixed orthodontic appliance was uniform in affecting all study subjects. Pain had a great contribution on changing dietary behavior of patients, and had a direct effect on physical properties of diet consumed by patients, since patients prefer softer food types and avoid harder ones ${ }^{(5)}$. That change in food preferences may cause a decrease in chewing behavior, which can cause an elevation in gingival inflammation (21). Another effect of pain and discomfort from using fixed orthodontic appliance is when practicing everyday oral preventive routine. Oral hygiene practice had the upper hand in controlling plaque and gingival health ${ }^{(22)}$. Although of different patients` attitude toward oral hygiene practice, but the design of the study eliminates these differences since it is a self-controlled study, so it measures changes on study subjects rather than differences between study subjects and controls. [4] Results of the present study may be included with other results of future studies that support no significant differences on gingival health among adolescent subjects with different BMI after ongoing under the course of fixed orthodontic treatment. The age of the patients may had an influence that provide higher immunological characteristics, which can compensate of the damaging effects of bacterial plaque on gingival health in spite of bad influence of thinness or obesity ${ }^{(23)}$. Each one of the previous four hypotheses needs more investigation to be proven, also each one needs to be edited and modified. Also new theories may be added when extensive researches about the same subject of this study are redone. The present study considered different than other studies because of categorizing early effect of fixed orthodontic treatment according to weight status of the patients on gingival health. Also what increase its individuality is it checks with patients after 2 weeks and then after 4 week from the first visit, which is very important to determine the true impact of orthodontic appliance away from patients` adaptation to pain and discomfort, which maximally recorded after 14 days ${ }^{(6)}$. Result of the present study revealed that there was an increasing in gingival index, a result that many researchers are in accordance with, especially within the period of four weeks or a month $(20,24,25)$. The present study revealed a significant elevation in gingival index at the $2^{\text {nd }}$ visit. Sudden impact of pain through the first period which led to a change in the form of the food to a softer and less fibrous composition ${ }^{(5)}$, less chewable diet leading to increased gingival inflammation (21). Addition of numerous components of fixed orthodontic appliance (brackets and auxiliaries) that increase the difficulty of the conventional oral hygiene practices (26), which led to rapid and effective plaque formation. Plaque bacteria which considered the main factor in increasing gingival inflammation $^{(3)}$, beside the traumatic action of the fixed orthodontic appliance components, like bands impinge on gingiva and over extended luting material over the gingiva ${ }^{(27)}$. The results showed that after the significant increase in gingival index on the $2^{\text {nd }}$ visit for all weight status groups there was a non significant increase for the underweight and overweight group, but kept its significant increase for normal weight status group. This may be related to the continuous exaggeration and consistent focusing of the immune system on the impact of fixed orthodontic appliance of normal weight group 
more than underweight and overweight groups (28).

\section{CONCLUSIONS}

There was a significant early impact of fixed orthodontic treatment on gingival health status; but there was no significant difference of that impact among weight status groups. Oral health preventive measures should be applied instantly and equally to all patients under fixed orthodontic treatment without taking their weight status in consideration.

\section{ACKNOWLEGMENT}

The authors would like to give deep appreciation, gratitude, and gratefulness to the administration, Dentists and orthodontists, medical staff, and all workers inside the specialized prosthodontics and orthodontic dental center in Bab-Almoatham /Baghdad /Iraq, for their contribution of the success of this study.

\section{REFERENCES}

1. Mitchell L. An introduction to orthodontics. 4th ed. United Kingdom: Oxford university press; 2013.

2. Klukowska M, Bader A, Erbe C, Bellamy P, White DJ, Anastasia MK, Wehrbein H. Plaque levels of patients with fixed orthodontic appliances measured by digital plaque image analysis. Am J Orthod Dentofacial Orthop. 2011; 139: 463-470

3. Marsh P, Lewis M, Rogers H, Williams D, Wilson M. Marsh and Martin`s Oral Microbiology. 6th ed. United Kingdom: Churchill Livingstone; 2017.

4. Shanbhog R, Raju V, Nandlal B. Correlation of oral health status of socially handicapped children with their oral heath knowledge, attitude, and practices from India. J Nat Sc Biol Med. 2014; 5(1):101-107.

5. Al Jawad FA. An investigation of the early effects of fixed orthodontic treatment on dietary intake and body weight in adolescent patients. United Kingdom, Queen Mary's School of Medicine and Dentistry/ University of London, PhD thesis dissertation; 2011.

6. Brown, D, Moerenhout, R. The pain experience and psychological adjustments to orthodontic treatment of preadolescents, adolescents and adults. Am J Orthod Dentofacial Orthop.1991; 100: 349-356.

7. Kalra S, Unni krishnan AG. Obesity in India: the weight of the nation. J Med Nutr Nutraceut. 2012; $1: 37-41$.

8. Deshpande NC, Amrutiya MR. Obesity and oral health - Is there a link? An observational study. J Indian Soc Periodontol. 2017; 21(3):229-233.

9. Al-Zahrani MS, Bissada NF, Borawskit EA. Obesity and periodontal disease in young, middle-aged, and older adults. J Periodontol. 2003; 74:610-5.

10. Scapoli L, Girardi A, Palmieri A, Martinelli M, Cura F, Lauritano D, Carinci F. Qualitative analysis of periodontal pathogens in periodontitis and gingivitis. J Biol Regul Homeost Agents. 2015; 29(3): 101-110.
11. Pihlstrom BL, Michalowicz BS, Johnson NW. Periodontal diseases. Lancet 2005; 366 (9499) : 1809-20.

12. Heitz-Mayfield LJ. Disease progression: identification of highrisk groups and individuals for periodontitis. $\mathrm{J} \mathrm{Clin} \mathrm{Periodontol}$ 2005; 32 (6):196-209.

13. Keller A, Rohde J F, Raymond K, Heitmann B L. Association between Periodontal Disease and Overweight and Obesity: A Systematic Review. J Periodontol. 2015, 86: 766-776.

14. Von Bremen J, Lorenz N, Ruf S. Impact of body mass index on oral health during orthodontic treatment: an explorative pilot study. Eur J Orthod. 2016; 38: 386-392.

15. World Health Organization. WHO expert consultation. Appropriate body mass index for Asian population and its implication for policy and intervention strategies. Lancet. 2004; 363:157-163.

16. De Onis M, Onyango AW, Borghi E, Siyam A, Nishida C, Siekmann J. Development of a WHO growth reference for school-aged children and adolescents. Bull World Health Organ. 2007; 85: 649-732.

17. WHO. Oral health surveys basic methods. 4th ed. World health organization. Geneva, Switzerland. 1997.

18. Loe H, Silness J. periodontal disease in pregnancy. acta odontol scand. 1963; 21: 533-51.

19. Loe, H. The gingival index, the plaque index system. J Periodontol. 1967; 38: 610-16.

20. Alidan E, AlRawi N. Oral health status among patients treated with fixed orthodontic appliance at different time intervals. Mustaniriya Dent J. 2016; 13 : 31-37.

21. Doi T, Hinode D, Nakae H, Yoshioka M, Matsuyama M, Iga H, Fukushima Y. Relationship between Chewing Behavior and Oral Conditions in Elementary School Children Based on the "Chewing 30" Program: An Intervention Study. J Dent Hlth. 2016; 66: 438-444.

22. Sreenlvasan P K, Prasad K V V, Javali B S. Oral health practice and prevalence of dental plaque and gingivitis among Indian adults. Clin Exp Dent Res. 2016; 2(1): 6-17.

23. Cole MF, Hsu SD, Baum BJ, et al. Specific and nonspecific immune factors in dental plaque fluid and saliva from young and old populations. Infect Immun. 1981; 31(3): 998-1002.

24. Naranjo AA, Trivino ML, Jaramillo A, Betancourth $\mathrm{M}$, Botero JE. Changes in the subgingival microbiota and periodontal parameters before and 3 months after bracket placement. Am J Orthod Dentofacial Orthop. 2006; 130: 17-22.

25. Lara-Carrillo E, Montiel-Bastida NM, Snchez-Pérez L, Alans-Tavira J. Changes in the oral environment during four stages of orthodontic treatment. Korean $\mathbf{J}$ Orthod. 2010; 40: 95-105.

26. Mount GJ, Hume WR. Preservation and restoration of tooth structure. 2nd ed. Queensland: Knowledge Books and Software; 2005. 
27. Kassab M M, Cohend E R. The etiology and prevalence of gingival recession. J Am Dent Assoc. 2003; 134(2): 220-225.

28. Niemanl DC, Nehlsen-Cannarellaz SL, Henson DA, Butterworth DE, Fagoagaz OR, Warren BJ, MK
Rainwater. Immune response to obesity and moderate weight loss. Int J Obes. 1996; 20: 353-360. 


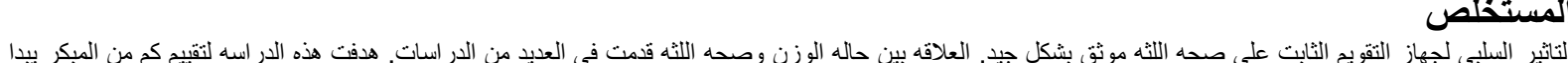

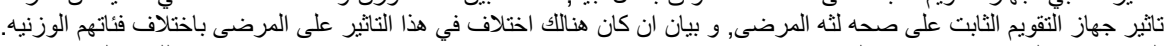

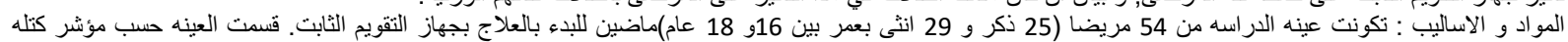

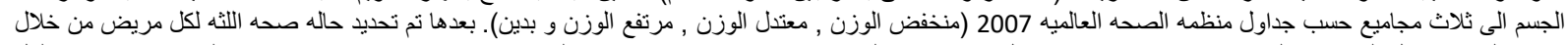

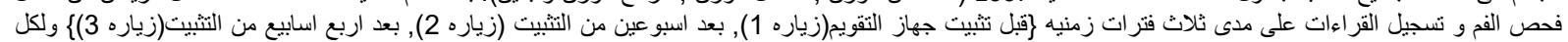

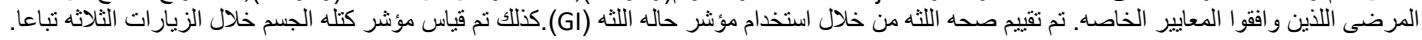

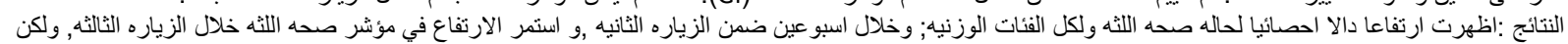

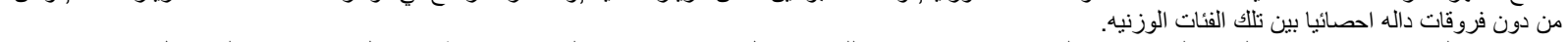

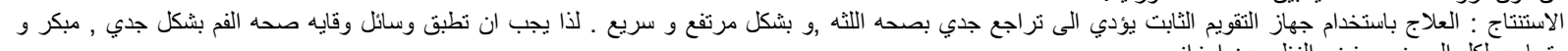

